\title{
ORIGINAL
}

\section{SIGNOS DE ALERTA DE TRASTORNOS ALIMENTARIOS, DE DEPRESIÓN, DEL APRENDIZAJE Y CONDUCTAS VIOLENTAS ENTRE ADOLESCENTES DE CANTABRIA (*)}

\author{
Horacio Paniagua Repetto (1) y Salvador García Calatayud (2) \\ (1) Centro de Salud «Dávila». Santander. \\ (2) Servicio de Pediatría. Hospital Universitario Marqués de Valdecilla. Santander.
}

\section{RESUMEN}

Fundamento: Los adolescentes presentan con relativa frecuencia trastornos de la conducta alimentaria, depresivos, del aprendizaje y conductas violentas, detectados tardíamente en muchas ocasiones. El objetivo de este trabajo fue investigar la presencia de signo de alerta ante estos trastornos entre los adolescentes y relacionarlos con el entorno social y familiar y los hábitos de vida.

Métodos: Estudio transversal descriptivo, mediante encuesta 2.178 adolescentes de 12 a 16 años, representativos de los adolescentes de Cantabria. Los signos de alerta se definieron a partir de los criterios de la Asociación Médica Americana y Asociación Americana de Psiquiatría.

Resultados: El 4,2\% de los adolescentes presenta signos de aler ta de trastornos del aprendizaje, asociados al sexo masculino, a la asistencia a colegios públicos y a repetir curso. El 4,3\% y el 10,2\% presenta signos de alerta de trastornos de la conducta alimentaria y trastornos depresivos, respectivamente, estando ambos asociados al sexo femenino. El 8,4\% tiene signos de alerta de conductas violentas, más frecuente en varones. Los signos investigados están distribuidos homogéneamente y aumentan con la edad. Están asociados de forma estadísticamente significativa con mayor consumo de tabaco, alcohol y drogas ilegales, episodios de embriaguez y determinado uso del tiempo libre por parte de los propios adolescentes, y con mayor consumo de sustancias adictivas por amigos y familiares.

Conclusiones: La prevalencia de signos de alerta en la adolescencia y sus consecuencias individuales y familiares, a corto y a largo plazo, justifican la detección en atención primaria, mediante cuestionarios sencillos que orienten al diagnóstico precoz.

Palabras clave: Adolescentes. Conducta alimentaria. Depresión. Aprendizaje. Violencia.

Correspondencia:

D Horacio Paniagua Repetto

Alta 46 A, Esc. «B», Piso $14^{\circ}$

39008 Santander

Correo electrónico: horaciopaniagua@ hotmail.com

\section{ABSTRACT}

\section{Warning Signs of Eating, Depressive, Learning Disorders and Violent Behavior among Teenagers in Cantabria, Spain}

Background: Teenagers relatively frequently suffer from eating, depressive learning and violent behavior disorders which are often detected late. This study was aimed at investigating the presence of warning signs among teenagers in view of these disorders and to relate them to the social and family environment and living habits.

Methods: A descriptive, cross-sectional study by means of a survey of 2,178 teenagers in the 12-16 age range, representative of the teenagers of Cantabria. The warning signs were defined based on the American Medical Association and American Psychiatric Association criteria.

Results: Warning signs of learning disorders related to the male gender, attending public schools and repeating a year were found among $4.2 \%$ of the teenagers. Warning signs of eating disorders and depressive disorders, both related to the female gender, were found respectively among $4.3 \%$ and $10.2 \%$ of the teenagers. A total of $8.4 \%$ showed warning signs of violent behavior, more often among males. The signs investigated are spread homogeneously, increasing with age, and are significantly related to a higher degree of smoking, drinking and illegal drugs, episodes of drunkenness and a certain use of free time on the part of the teenagers, with a higher degree of free time on he partof the teengers, wit a higher degee of bers.

Conclusions: The routine assessment during the teen years of warning signs of having the disorders studied would make their early detection possible.

Key words: Adolescent. Eating behavior. Depression. Learning. Violence.

(*) Estudio financiado por la Fundación «E. Sánchez Villares» de la Sociedad de Pediatría de Asturias, Cantabria y Castilla León. II Convocatoria de Ayudas a la Investigación Clínica y Epidemiológica en Pediatría. (1999). 


\section{INTRODUCCIÓN}

La adolescencia es una etapa de la vida en la que pueden observarse trastornos de la conducta alimentaria, depresivos, del aprendizaje y conductas violentas, detectados tardíamente en muchas ocasiones. Diversos estudios poblacionales investigan su prevalencia ${ }^{1-6} y$ otros intentan detectar, a través de la presencia de diversos signos, a los adolescentes con riesgo de padecer algunas de estas situaciones ${ }^{7,8}$. También se han delimitado patrones o perfiles diferenciados relacionados con estos trastornos y que pudieran ser predictivos de estas patologías en la adolescencia ${ }^{9-11}$.

De no mediar un diagnóstico precoz y un tratamiento oportuno, estas situaciones perdurarán e incrementarán su sintomatología a lo largo de la adolescencia, provocando dificultades personales y familiares que pueden mantenerse en la vida adulta. La detección en atención primaria, a través de una valoración rutinaria de signos de sospecha de padecer alguno de los trastornos citados, permitiría actuaciones diagnosticas y terapéuticas tempranas que redundarían en un mejor pronóstico a corto y largo plazo.

Los trastornos de la conducta alimentaria se observan con frecuencia en el mundo occidental y desarrollado, habiéndose producido un aumento de su prevalencia en las últimas décadas ${ }^{11}$. Otros autores relacionan el incremento a un cambio en el patrón de presentación de los trastornos mentales ${ }^{12}$. A pesar de no estar resuelta esta controversia ${ }^{13}$ los trastornos de la conducta alimentaria, anorexia y bulimia, son una causa frecuente de enfermedad crónica en mujeres durante la adolescencia11, con sintomatología que está presente en algunos casos en los primeros años de esta época de la vida ${ }^{14}$. En el aumento de la incidencia y prevalencia de los trastornos de la conducta alimentaria estarían involucrados, además de la mejoría en los procedimientos diagnósticos, el cambio en los valores estéticos de la sociedad, con un determinado culto al cuerpo y la influencia que los medios de comunicación puedan ejercer al relacionar la perfección corporal con éxito social, además de otros factores sociosanitarios ${ }^{13}$. Existen diversos medios para evaluar los trastornos de la conducta alimentaria, como el EAT y EDI ${ }^{15,16}$ y hay autores que proponen para atención primaria, cuestionarios de dos y cuatro preguntas que permitirían sospechar hallarse ante estos trastornos ${ }^{17,18}$. En estos tests rápidos se investiga fundamentalmente la imagen corporal, hábitos alimenticios y realización de dietas de adelgazamiento. En relación a los trastornos de la conducta alimentaria la insatisfacción corporal se ha identificado como uno de los factores de riesgo ${ }^{19}$ y las dietas y el desorden alimentario como conductas de riesgo ${ }^{20}$. También se han analizado conductas anoréxicas entre adolescentes y se ha observado que una amplia mayoría de las mujeres preferiría estar más delgada y perder peso para sentirse con una figura corporal ideal, y la mitad de ellas reconocen haber hecho régimen alimenticio; los valores encontrados en varones se reducen a más de la mitad $^{21}$. Un diagnóstico temprano y un tratamiento adecuado condicionan una mejor evolución de estos trastornos, por lo que el personal sanitario en atención primaria debería investigar los comportamientos de riesgo y sus signos de alarma, y de esta forma llegar a un diagnóstico precoz a través de una investigación más profunda ${ }^{22}$.

Los trastornos emocionales y el humor deprimido transitorios, se detectan con frecuencia entre los adolescentes. La presencia de trastornos depresivos mayores en estas edades fue puesta en duda durante mucho tiempo y desde hace pocas décadas se acepta su diagnóstico en la adolescencia, lo que ocurre con relativa frecuencia2. Los trastornos depresivos mayores en los adolescentes se asocian con mayor consumo de tabaco, alcohol y drogas ilegales y con actividades sexuales de riesgo 23,24 , ideas suicidas e intentos de suicidio ${ }^{25,26}$. La depresión mayor se ha identificado como la tercera causa de 
trastornos psiquiátricos, después del insomnio y la distimia, en la población general adolescente española a los 18 años, según criterios DSM-III-R ${ }^{5}$, y es el segundo diagnóstico en frecuencia, con criterios CIE-10, entre aquéllos que solicitan tratamiento psicológico o son derivados para ello ${ }^{6}$. Los adolescentes con depresión mayor a los 18 años de edad, refieren mayoritariamente sintomatología depresiva antes de los 14 años y una tercera parte de ellos fueron diagnosticados en esta edad 9 . Los síntomas depresivos en la adolescencia son factores de riesgo para padecer trastornos mentales en jóvenes adultos, entre ellos la depresión mayor ${ }^{27}$. Existe la recomendación de investigar rutinaria y anualmente síntomas depresivos en la adolescencia ${ }^{28,29}$ y en adultos se utilizan cuestionarios cortos para realizar cribado de estos trastornos $^{30}$.

Las dificultades escolares ${ }^{31}$ o fracaso escolar, definido como la no consecución de los objetivos educativos esperados ${ }^{32}$, es una situación frecuente en la adolescencia. Según datos oficiales afecta a una cuarta parte de esta población española, cifras que son superiores a otros países europeos ${ }^{33}$. Los adolescentes deberían ser examinados periódicamente en busca de problemas académicos, como la disminución o falta de progreso escolar, y también sobre signos de rechazo hacia la escuela, como son los novillos o ausencias repetidas ${ }^{34}$. Desde un punto de vista sanitario, hay un grupo de trastornos que pueden provocar un fracaso escolar, como son los trastornos del aprendizaje, que incluye a la dislexia, el trastorno de la escritura y del cálculo y otra entidad que puede estar asociada, como los déficits de atención y la hiperactividad ${ }^{35}$. Ambas situaciones deberían ser investigadas por el personal sanitario ante una dificultad escolar referida por un adolescente ${ }^{34}$.

La violencia en la adolescencia, en aumento en las últimas décadas, es considerada como un problema de salud pública en determinados países como Estados Uni- $\operatorname{dos}^{36}$. Su prevalencia en adolescentes europeos, aunque menos estudiada, es menor que la norteamericana ${ }^{37}$. Los análisis de la violencia entre los jóvenes españoles muestran que por un lado pueden ser víctimas de comportamientos violentos por su entorno social, su familia y por desconocidos, y por otro, ser parte activa a través de comportamientos agresivos, con mayor frecuencia con sus pares y en segundo lugar con sus padres $^{4}$. La violencia, junto a otros factores, está relacionada con la alta morbimortalidad en estas edades ${ }^{38}$ y asociada a un mayor consumo de drogas legales e ilegales, prácticas sexuales de riesgo, e ideas y conductas suicidas $^{39,40}$. Entre los adolescentes se pueden identificar signos que alerten sobre la presencia de estas conductas e intentar realizar actuaciones preventivas entre ellos ${ }^{41}$. En atención primaria, la utilización de cuestionarios simples referidos a situación escolar y uso de drogas en la adolescencia, pudieran ser predictivos de conductas violentas posteriores ${ }^{42}$.

El objetivo de este estudio es investigar la presencia de signos de alerta de trastornos de la conducta alimentaria, depresivos, del aprendizaje, y de conductas violentas entre los adolescentes y relacionarlos con el consumo de alcohol, tabaco y drogas no legales, así como con sus hábitos de vida y el entorno.

\section{SUJETOS Y MÉTODOS}

\section{Población de estudio}

El estudio, transversal y descriptivo, fue realizado entre la población de 12 a 16 años de la Comunidad Autónoma de Cantabria, constituida por 25.200 adolescentes, todos ellos escolarizados en Educación Secundaria Obligatoria. Se estimó un tamaño muestral de 2.112 encuestas para $p=0,40, d=2 \%$ y $\alpha=0,05$. Dada la relación de alumnos por aula en esta Comunidad y previendo negativas a participar, absentismo escolar y des- 
cartes por errores en la cumplimentación de la encuesta, se decidió encuestar a los alumnos de 92 aulas. Teniendo en cuenta la distribución de la población en áreas rurales y urbanas (1/2), se dividieron éstas según pertenecieran a núcleos de población menores o mayores a 10.000 habitantes, obteniéndose 32 aulas rurales y 60 aulas urbanas. Además, las aulas se clasificaron en públicas y concertadas según la proporción existente en esta comunidad entre estas dos modalidades de educación (7/3).

Las aulas se repartieron aleatoriamente respetando los criterios de estratificación. Previa autorización de la Dirección General de Educación del Gobierno de Cantabria y de los colegios seleccionados, y después de la realización de un estudio piloto, el trabajo de campo se llevó a cabo entre el 20 de noviembre de 1999 y el 31 de enero de 2000.

\section{Descripción de la muestra}

Se analizaron las encuestas de 2.178 adolescentes (50,1\% varones, $49,9 \%$ mujeres), con edades comprendidas entre los 12 y 16 años, y distribuidos por edades según los siguientes porcentajes: 12 años: 19,29 \%, 13 años: $21,18 \%, 14$ años: 24,48 \%, 15 años: $22,42 \%$ y 16 años: $12,53 \%$. El 66,9\% reside en zonas urbanas y el resto en rurales, concurriendo el $69,4 \%$ de los encuestados a colegios públicos y el resto a enseñanza concertada.

\section{Cuestionario}

El cuestionario, diseñado específicamente para este estudio, y basado en el cuestionario FRISC $^{43}$, constaba de 88 preguntas, de las cuales 46 eran con opción doble y el resto múltiple con una única respuesta válida. Fue cumplimentado por los alumnos en sus aulas de forma anónima y voluntaria, con la presencia del personal docente habitual y de un investigador. Se garantizó previamente a los adolescentes la confidencialidad de los datos.
Se recogieron variables demográficas, consumo de drogas legales e ilegales entre los adolescentes, sus amigos y familiares. También diferentes aspectos sobre ocio y tiempo libre, variables psicoafectivas, alimentarias, relacionadas con el aprendizaje y con conductas violentas. No hubo negativas por parte de los alumnos para ser encuestados y se excluyeron las encuestas pertenecientes a adolescentes con edades fuera de rango, o con errores de cumplimentación.

\section{Definición de signos de alerta}

Para considerar a un adolescente como portador de signos de alerta frente a los trastornos a investigar, se definieron las condiciones que debía reunir para ser incluido en cada grupo, a partir de diversos parámetros encontrados en la literatura y que fueron los siguientes:

Signos de alerta de trastornos de la conducta alimentaria:

La Guía de las Actividades Preventivas en el Adolescente de la Asociación Médica Americana (GAPA-AMA) ${ }^{44}$ recoge que conductas de mantenimiento crónico de dietas y mala imagen corporal deberían alertar al médico para realizar una valoración más profunda con el fin de descartar el diagnóstico de anorexia nerviosa o bulimia. Se definieron como signos de alerta los siguientes:

- Conductas de mantenimiento crónico de dieta (tres o más dietas de adelgazamiento el último año).

- Mala imagen corporal.

Signos de alerta de trastornos depresivos:

En relación a los signos de alerta de trastornos depresivos, se definieron como tales a 
los dos primeros síntomas del diagnóstico de episodio depresivo mayor del DSM-IV ${ }^{45}$, recordando que uno de ellos debe estar siempre presente en el diagnóstico de trastorno depresivo mayor. Fueron investigados los siguientes:

- Humor deprimido o irritable.

- Pérdida de interés o placer frente a las actividades cotidianas.

Signos de alerta de trastornos primarios de aprendizaje:

La GAPA-AMA ${ }^{34}$ indica que adolescentes con conductas establecidas de rechazo escolar, problemas de comportamiento y falta o declinación del progreso académico pueden ser portadores de un trastorno del aprendizaje. Fueron investigados en este estudio:

- Progreso escolar crónicamente bajo.

- Faltas repetidas a la escuela.

- Dificultades de comportamiento.

\section{Signos de alerta de conducta violenta:}

Las actividades violentas descritas con mayor frecuencia en las que participan los jóvenes, son las peleas y agresiones entre amigos y/o compañeros de estudios o trabajo, seguidas por agresiones en el seno familiar y en tercer lugar con extraños ${ }^{4}$. Se consideraron signos de alerta:

- Haber llegado a la violencia con familiares o amigos.

- Estar envuelto a menudo en peleas o riñas.

Los signos de alerta definidos fueron trasladados a doce preguntas, en un lenguaje coloquial y comprensible para los adoles- centes, como se muestra en el Anexo 1. Para considerar a un adolescente portador de un signo de alerta debía contestar afirmativamente a todas las cuestiones relativas al signo investigado.

\section{Análisis de los datos}

Las encuestas fueron leídas mediante un lector óptico de marcas y se realizó una corrección manual de dicha lectura. Los resultados fueron codificados y grabados sobre una base de datos DBASE IV, tras lo cual se realizó una depuración lógica de los registros obtenidos. Se hicieron análisis univariante y bivariante para la comparación de variables cualitativas mediante la prueba de la $\chi^{2}$, utilizando para todo ello el paquete estadístico SPSS v9.0.

\section{RESULTADOS}

\section{Signos de alerta de trastornos de la conducta alimentaria}

El 22,9\% de los adolescentes (IC 95\%: 21,4-25,2) refiere mala imagen corporal y el $6 \%$ (IC 95\%: 4,4-9,1) ha realizado tres o más dietas de adelgazamiento el último año. El 4,3\% (IC 95\%: 3,5-5,2) de los encuestados cumple los dos signos de alerta y a los 15 años el 5,1\% (IC 95\%: 3,2-7,3). Están asociados significativamente con el sexo femenino (9:1) ( $<<0,0001)$ y aumenta con la edad (tabla 1), con mayor consumo de alcohol $(p<0,05)$ y con episodios de embriaguez $(\mathrm{p}<0,01)$. Su distribución es homogénea en cuanto, no se observan diferencias por localidad de residencia urbana o rural, asistencia a colegio público o concertado o nivel de estudio de los padres. Al analizar las horas de deporte extraescolar que realizan semanalmente, no se observan diferencias significativas, comparando tanto con la población estudiada en general como con las mujeres que no presentan el riesgo. Las preferencias en el uso del tiempo libre de los adolescentes en riesgo se reflejan en la tabla 2 . 
Anexo 1

Relación de preguntas realizadas a los adolescentes para investigar los signos de alerta definidos

\begin{tabular}{|c|c|}
\hline Signos de alerta & Preguntas \\
\hline Trastornos del aprendizaje & $\begin{array}{l}\text { Tu rendimiento escolar es: } \\
\text { - Bueno } \\
\text { - Malo } \\
\text { Te «corres» las clases a menudo?: } \\
\text { - No } \\
\text { - Sí } \\
\text { ¿Te riñen tus profesores frecuentemente por mal comportamiento en clase?: } \\
\text { - No } \\
\text { - Sí }\end{array}$ \\
\hline Trastornos de la conducta alimentaria & $\begin{array}{l}\text { ¿Has hecho alguna vez dietas de adelgazamiento? } \\
\text { - No } \\
\text { - Sí, } 1 \text { ó } 2 \text { veces el último año } \\
\text { - Sí, más de } 2 \text { veces el último año } \\
\text { ¿Estás contento/a con tu cuerpo? } \\
\text { - No } \\
\text { - Sí }\end{array}$ \\
\hline Trastornos depresivos & $\begin{array}{l}\text { ¿Estás triste o desesperado/a con frecuencia? } \\
\text { - No } \\
\text { - Sí } \\
\text { ¿Has perdido interés por las cosas que siempre te gustaban? } \\
\text { - No } \\
\text { - Sí }\end{array}$ \\
\hline Conductas violentas & $\begin{array}{l}\text { ¿Con tus familiares o amigos has llegado alguna } \\
\text { vez a la violencia? } \\
\text { - No } \\
\text { - Sí } \\
\text { ¿Te ves envuelto a menudo en peleas o riñas? } \\
\text { - No } \\
\text { - Sí }\end{array}$ \\
\hline
\end{tabular}

\section{Signos de alerta de trastornos depresivos}

El 19,7\% (IC 95\%: 18,4-21,8) refiere tener un humor deprimido o irritable con frecuencia y el 24,6\% (IC 95\%: 23,1-26,4) ha perdido interés por las cosas que previamente le atraían. El 10,2 \% (IC 95\%: 8,9-11) responde afirmativamente a las dos cuestiones, por lo que estarían incluidos en el grupo de adolescentes con riesgo de trastornos depresivos. Se asocia estadísticamente con el sexo femenino $(\mathrm{p}<0,005)$ y con mayor edad (tabla $1)$, con un mayor consumo de tabaco $(\mathrm{p}<0,0001)$, alcohol $(\mathrm{p}<0,001)$ y drogas ilegales $(\mathrm{p}<0,0001)$ y con un mayor número de episodios de embriaguez en los 6 meses previos a ser encuestados $(p<0,0001)$. El resto de las variables muestran una distribución homogénea, ya que no hay diferencias por localidad de residencia, tipo de colegio al que asisten o nivel de estudios de los padres. El uso del tiempo libre y de ocio de los adolescentes en riesgo se observa en la tabla 2.

\section{Signos de alerta de trastornos de aprendizaje}

El 12,4\% (IC 95\%: 11,2-14,7) de los adolescentes hace novillos con frecuencia, el 20,2\% (IC 95\%: 18,1-22,6) refiere dificult ades de comportamiento en el colegio y el $30,9 \%$ (IC 95\% :29,2-33,4) reconoce mal adimiento escolar. Cumplen los tres criterios de signos de alerta definidos el 4,2\% (IC 95\%: 3,4-5,1) de los adolescentes encuestados. La prevalencia de este signo de alerta aumenta con la edad y se asocia significativamente con el sexo masculino ( $\mathrm{p}<0,001)$ (tabla $1)$, con repetir curso ( $p<0,0001)$, con la concurrencia a colegios públicos $(8 / 2)$ $(p<0,0001)$, con la creencia de que el tabaco $(\mathrm{p}<0,0001)$ y el alcohol $(\mathrm{p}<0,001)$ no son drogas, con mayor consumo de tabaco $(\mathrm{p}<0,0001)$, alcohol $(\mathrm{p}<0,0001)$ y drogas ilegales $(\mathrm{p}<0,0001)$ y mayor número de episodios de embriaguez en sus vidas $(\mathrm{p}<0,0001)$ y en los 6 meses previos a ser encuestados 
Tabla 1

Distribución por edades y sexo de los adolescentes con signos de alerta de trastornos de la conducta alimentaria, depresivos, del aprendizaje y conductas violentas

\begin{tabular}{|c|c|c|c|c|c|c|c|c|}
\hline $\mathbf{n}=\mathbf{2 . 1 7 8}$ & \multicolumn{2}{|c|}{$\begin{array}{c}\text { Trastornos conducta } \\
\text { alimentaria }\end{array}$} & \multicolumn{2}{|c|}{ Trastornos depresivos } & \multicolumn{2}{c|}{ Trastornos aprendizaje } & \multicolumn{2}{c|}{ Conductas violentas } \\
\hline Edad & varones & mujeres & varones & mujeres & varones & mujeres & varones & mujeres \\
\hline 12 & $0,24 \%$ & $1,19 \%$ & $2,88 \%$ & $2,64 \%$ & $0,00 \%$ & $0,00 \%$ & $3,59 \%$ & $2,39 \%$ \\
\hline 13 & $0,87 \%$ & $3,48 \%$ & $3,05 \%$ & $4,79 \%$ & $1,09 \%$ & $0,65 \%$ & $4,59 \%$ & $2,18 \%$ \\
\hline 14 & $0,38 \%$ & $4,17 \%$ & $4,74 \%$ & $6,64 \%$ & $2,67 \%$ & $1,53 \%$ & $5,88 \%$ & $2,66 \%$ \\
\hline 15 & $0,41 \%$ & $4,76 \%$ & $3,71 \%$ & $6,80 \%$ & $4,14 \%$ & $2,90 \%$ & $6,82 \%$ & $3,51 \%$ \\
\hline 16 & $0,36 \%$ & $5,84 \%$ & $7,69 \%$ & $10,62 \%$ & $8,06 \%$ & $1,83 \%$ & $6,99 \%$ & $3,68 \%$ \\
\hline
\end{tabular}

Tabla 2

Asociación estadística entre los signos de alerta estudiados y el uso que hacen los adolescentes de su tiempo libre y de ocio $(n=2.178)$

\begin{tabular}{|l|l|c|c|}
\hline Signos de alerta & Variables asociadas & $\mathbf{n}$ & $\mathbf{p}$ \\
\hline \multirow{4}{*}{ Conductas violentas } & Salir con amigos a diario o semanalmente & 117 & $<0.0001$ \\
& Ir a la discoteca semanalmente & 118 & $<0.0001$ \\
& Usar ordenador/videoconsola semanalmente & 223 & $<0.0001$ \\
& Nunca leer libros & 82 & $<0.0001$ \\
& Nunca ir de excursión & 58 & 0.002 \\
\hline \multirow{3}{*}{ Trastornos de la conducta } & & & \\
alimentaria & Escuchar música diariamente & 208 & 0.014 \\
\hline \multirow{3}{*}{ Trastornos del aprendizaje } & Ir a la discoteca semanalmente & 80 & $<0.0001$ \\
& Ir de copas semanalmente & 61 & 0.001 \\
\hline & Salir con amigos semanalmente & 88 & $<0.0001$ \\
& Salir de copas semanalmente & 56 & $<0.0001$ \\
& Nunca lectura revistas/comics & 36 & 0.002 \\
\hline Trastornos depresivos & Nunca lectura libros & 69 & $<0.0001$ \\
& Nunca práctica deporte & 31 & 0.01 \\
\hline
\end{tabular}

$(\mathrm{p}<0,0001)$. La distribución es homogénea entre adolescentes rurales y urbanos y por nivel de estudios de los padres. El uso del tiempo libre que hacen estos adolescentes se relaciona con una mayor actividad social y menor actividad intelectual y deportiva (tabla 2).

\section{Indicadores de alerta de conducta violenta}

El 17,3\% (IC 95\%: 16,2-19,1) se ve envuelto con frecuencia en peleas o riñas; el
26,5\% (IC 95\%: 25,6-29,2) ha llegado alguna vez a la violencia con amigos o familiares. El 8,4 \% (IC 95\%: 7,2-9,5) responde afirmativamente a las dos cuestiones. Está asociado significativamente con el sexo masculino $(p<0,0001)$ y aumenta con la edad (tabla 1), con la creencia de que tabaco $(\mathrm{p}<0,001)$ y alcohol $(\mathrm{p}<0,0001)$ no son drogas, con mayor consumo de tabaco $(p<0,001)$, alcohol $(p<0,0001)$ y drogas ilegales $(p<0,0001)$ y con mayores episodios de embriaguez en sus vidas $(\mathrm{p}<0,0001)$ y los 6 meses previos a la encuesta $(p<0,0001)$. El 
resto de la distribución es homogénea, ya que no hay diferencias estadísticas por localidad de residencia, tipo de colegio público o concertado o nivel de estudios de los padres. El uso del tiempo libre por estos adolescentes se refleja en la tabla 2.

Los cuatro signos de alerta estudiados se asocian estadísticamente a un mayor consumo de drogas legales e ilegales entre los amigos de los adolescentes en riesgo y el signo de alerta de conductas violentas con un mayor consumo de alcohol entre padres y hermanos de los adolescentes (tabla 3). El análisis multivariante de estos factores asociados no ofreció datos significativos.

\section{Asociación entre los diferentes signos de alerta}

Los signos de alerta de trastornos de la conducta alimentaria y de conductas violentas se asocian estadísticamente, cada uno, con el resto de los signos de alerta estudia- dos. Los signos de trastornos de la conducta alimentaria se asocian con trastornos depresivos $(p<0,0001)$, del aprendizaje $(\mathrm{p}<0,0001)$, y de conductas violentas $(\mathrm{p}<0,002)$. Los signos de conductas violentas lo hacen con trastornos del aprendizaje $(\mathrm{p}<0,0001)$, trastornos de la conducta alimentaria y depresivos $(\mathrm{p}<0,0001)$.

El resto de los signos de alerta tienen menor asociación; así los depresivos y los del aprendizaje lo están con los signos de trastornos de la conducta alimentaria y de conductas violentas.

\section{DISCUSIÓN}

La población de adolescentes de este estudio representa a la práctica totalidad de los individuos de 12 a 16 años ya que a estas edades la colegiación es obligatoria. Diversos autores validan cribados rápidos en atención primaria con dos y cuatro preguntas para detectar probables trastornos alimenta-

Tabla 3

Asociación estadística entre los signos de alerta estudiados y el consumo de tabaco, alcohol y drogas ilegales en el entorno del adolescente. $\mathbf{N}=\mathbf{2 . 1 7 8}$

\begin{tabular}{|l|l|c|c|}
\hline Signos de alerta & Variables asociadas & n & p \\
\hline \multirow{4}{*}{ Conductas violentas } & Padre bebedor & 233 & 0.01 \\
& Madre bebedora & 140 & 0.006 \\
& Padre fumador & 180 & 0.05 \\
& Hermanos bebedores & 128 & 0.001 \\
& Amigos fumadores & 175 & $<0.0001$ \\
& Amigos bebedores & 219 & $<0.0001$ \\
& Amigos consumidores drogas ilegales & 52 & $<0.0001$ \\
\hline \multirow{5}{*}{ Trastornos de la conducta } & Madre bebedora & 83 & 0.001 \\
& Amigos fumadores & 130 & $<0.0001$ \\
& Amigos bebedores & 152 & $<0.0001$ \\
\hline \multirow{3}{*}{ Trastornos } & Amigos consumidores de drogas ilegales & 44 & $<0.0001$ \\
del aprendizaje & Amigos fumadores & 70 & $<0.0001$ \\
\hline Trastornos depresivos & Amigos bebedores & 81 & $<0.0001$ \\
& Amigos consumidores de drogas ilegales & 36 & $<0.001$ \\
\hline & Amigos fumadores & 27 & $<0.0001$ \\
& Amigos bebedores & 34 & $<0.0001$ \\
\hline
\end{tabular}


$\operatorname{rios}^{17,18}$. Los adolescentes con probable riesgo de padecer trastornos de la conducta alimentaria se presentan con una frecuencia cuatro veces mayor que las cifras de prevalencia de anorexia nerviosa y bulimia encontradas en Navarra entre las adolescentes de 12 a 21 años ${ }^{14}$. Al analizar los valores encontrados a los 15 años en este estudio se observa que triplican la prevalencia encontrada por Morandé et $\mathrm{al}^{1}$ a esta edad, teniendo en cuenta los criterios de diagnóstico. Las variables que acompañan a estos adolescentes son similares a las de los portadores de estas enfermedades. Son mayoritariamente mujeres, coincidiendo con la prevalencia por sexo ${ }^{1,14}$, como con la distribución homogénea de la muestra en los estratos sociales, residenciales y educativos, referida en la mayoría de los estudios actuales ${ }^{11}$. Se observa un mayor consumo de alcohol, como es referido en los trastornos de la conducta alimentaria, fundamentalmente bulimia ${ }^{46}$. El incremento en la práctica de actividad física sistemática, presente en la mayoría de casos antes de iniciarse la anorexia nerviosa, no se observa entre estos adolescentes ${ }^{47}$. Este signo de alerta se asocia con el de riesgo de trastornos depresivos, de forma similar a la relación descrita entre anorexia y depresión ${ }^{48}$.

La Academia Americana de Pediatría recomienda realizar preguntas de rutina sobre depresión en la historia clínica del adolescente $^{28}$, y la Asociación Médica Americana aconseja investigar anualmente síntomas de depresión en estas edades ${ }^{29}$. En el diagnóstico de depresión, la disforia y la anhedonia son criterios fundamentales, acompañados de otros síntomas ${ }^{45}$. Hay cuestionarios validados de dos preguntas sobre trastornos del humor y anhedonia, en atención primaria de adultos ${ }^{30}$. La frecuencia de adolescentes que estarían en riesgo de padecer trastornos afectivos es cinco veces superior a la frecuencia de depresión mayor en adolescentes urbanos de 13-14 años en España $^{2}$. Son mayoritariamente mujeres, como se observa en la depresión mayor a estas edades, y tienen un mayor consumo de tabaco, alcohol y drogas ilegales, como se describe en estos trastornos ya diagnostica$\operatorname{dos}^{23,49}$.

Los trastornos del aprendizaje no tienen una prevalencia determinada en la adolescencia, siendo estimada en valores que van desde el 3 al 10\%, según la definición y criterios aplicados por diversos autores ${ }^{34}$. Los adolescentes estudiados que estarían en riesgo de padecer un trastorno del aprendizaje están por debajo de la media de la prevalencia supuesta referida. Son mayoritariamente varones como se refiere en estos trastor$\operatorname{nos}^{34}$. Al relacionar el consumo de sustancias y el uso del tiempo libre de los adolescentes con signos de alerta, con trabajos que investigan el fracaso escolar, se observa en ellos un mayor consumo de tabaco, alcohol y drogas ilegales y un determinado uso del tiempo libre, más proclive a mayores actividades sociales y menores actividades intelectuales y deportivas ${ }^{3,50}$.

La prevalencia de comportamientos agresivos y disociales en la adolescencia varía entre el 1,5 y el 8,7 \%, según las fuentes de información utilizada y la edad de los encuestados $^{51}$. Los valores encontrados de adolescentes con probables conductas violentas de este estudio son similares a la mayor prevalencia referida. Se observa con más frecuencia en adolescentes varones, de igual manera a lo referido por Laespada y Salazar ${ }^{4}$. En relación al sexo, existe un sesgo, ya que no se interroga sobre formas verbales de violencia y conductas de aislamiento y segregación social, formas más frecuentes de ejercer la violencia por mujeres adolescentes ${ }^{52}$. Las conductas violentas o delictivas en la adolescencia se asocian con el consumo de drogas legales e ilegales ${ }^{53}$, observándose dicha relación entre los adolescentes con este signo de alerta. La distribución de estos adolescentes en riesgo es homogénea en la sociedad, no habiendo encontrado diferencias con respecto a una mayor prevalencia urbana, como refieren otros autores $^{51}$. 
El mayor consumo de sustancias adictivas está presente en todos los signos de alerta estudiados. El entorno social de los adolescentes, dado por los amigos, es mayor consumidor de tabaco, alcohol y drogas ilegales en todos los signos de alerta investigados. Se observa, por otra parte, mayor consumo de alcohol en el entorno familiar, padres y hermanos, de los adolescentes con probables conductas violentas.

Este estudio, al ser transversal y descriptivo, no da lugar a conclusiones que permitan relacionar a los adolescentes con signos de alerta con enfermedades futuras, pero es de destacar que los cuatro signos de alerta estudiados están asociados estadísticamente a variables como el sexo y otras que coinciden con las presentes en cada uno de estos trastornos o enfermedades ya diagnosticados. Su prevalencia en la adolescencia y sus consecuencias individuales y familiares, a corto y a largo plazo, justifican la detección en atención primaria, mediante cuestionarios sencillos, de signos de alerta que orienten a sus diagnósticos precoces.

\section{AGRADECIMIENTOS}

Deseamos expresar nuestro agradecimiento al Dr. Carlos Redondo Figuero por su asesoramiento estadístico para la realización del presente trabajo.

\section{BIBLIOGRAFÍA}

1. Morandé G, Casas J, Celada. Prevalence of eating disorders in Spanish school-age population. J. Adolesc Health 1999; 24: 212-9.

2. Canals J, Martí-Henneberg C, Fernández-Ballart J, Domenech E. A longitudinal study of depression in an urban Spanish pubertal population. Eur Child Adolesc Psychiatry 1995; 4: 102-11.

3. Ojembarrena Martínez E, Fernández de Pinedo Montoya R, Gorostiza Garay E, Lafuente Mesanza P, Lizárraga Azparren MA. Fracaso escolar en adolescentes y factores sociosanitarios asociados. An Esp Pediatr 2002; 56: 416-424.
4. Laespada MT, Salazar L. El joven como objeto y sujeto de comportamientos violentos. En: Elzo J, Orizo FA, González-Anleo J, González Blasco P, Laespada MT, Salazar L (eds.). Jóvenes españoles 99. Fundación Santa María;1999. p.388-400.

5. Canals J, Doménech, Carbajo G, Bladé J. Prevalence of DSM-R and ICD-10 psychiatric disorders in a Spanish population of 18 years olds. Acta Psychiatr Scand 1997; 96: 287-94.

6. Aláez Fernández M, Martínez Arias R, RodríguezSutil C. Prevalencia de trastornos psicológicos en niños y adolescentes, su relación con la edad y el género. Psicothema 2000; 4: 525-32.

7. Elster AB, Kuznets NJ. Guía de la AMA para actividades preventivas en el adolescente (GAPA). Recomendaciones y fundamentos. Madrid: Díaz de Santos; 1995.

8. American School Health Association. The National Adolescent Student Health Survey: A Report on the Health of America's Youth. Kent OH: American School Health Association; 1989.

9. Canals J, Domènech-Llaberia E, Fernández-Ballart J, Martí-Hennenberg C. Predictors of depression at eighteen. A 7-year follow-up study in a Spanish nonclinal population. Europ Child Adolesc Psychiatry 2002; 5: 226-33.

10. Bragado C, Bersabé R, Carrasco I. Factores de riesgo para los trastornos conductuales, de ansiedad, depresivos y de eliminación en niños y adolescentes. Psicothema 1999; 4. 939-56.

11. Morandé G, Casas J. Trastornos de la conducta alimentaria en adolescentes. Anorexia nerviosa, bulimia y cuadros afines. Pediatr Integral 1997; 2: 243-60.

12. Pedreira Massa JL. Trastornos de la conducta alimentaria en adolescentes: ¿Una enfermedad? ¿Una epidemia?. An Esp Pediatr 1998; 49: 434.

13. Guerro-Prado D, Barjau Romero JM, Chinchilla Moreno A. Epidemiología de los trastornos de la conducta alimentaria e influencia mediática: una revisión de la literatura. Actas Esp Psiquiatr 2001; 29: 403-10.

14. Pérez-Gaspar M, Gual P, de Irala-Estévez J, Martínez-González MA, Lahortiga F, Cervera S. Prevalencia de trastornos de conducta alimentaria en las adolescentes navarras. Med Clin (Barc) 2000; 114: 481-6.

15. Garner DM, Garfinkel PE. The Eating Attitudes Test: an index of the symptoms of anorexia nervosa. Psychol 1979; 9: 273-9. 
16. Garner DM, Olmsted MO, Polivy J. Development and validation of a multidimensional inventory for anorexia nervosa and bulimia. Int $\mathbf{J}$ Eat Disord $1983 ; 2: 15-34$.

17. Freund KM, Graham SM, Lesky L, Moskowitz MA. Detection of bulimia in a primary care setting. J Gen Intern Med 1993; 8: 236-42.

18. Anstine D, Grinenko D. Rapid screening for disorder eating in college-aged females in the primary care setting. J Adolescent Health 2000; 26: 338-42.

19. Ballester F, de Gracia Blanco M, Patiño Masó J, Suñol Gurnés C, Ferrer Avellí M. Actitudes alimentarias y satisfacción corporal en adolescentes: un estudio de prevalencia. Actas Esp Psiquiatr 2002; 30: 207-312.

20. Morandé G. La insatisfacción corporal como factor de riesgo en el desarrollo de la anorexia nerviosa del niño y el adolescente. Revista de Psiquiatría y Psicología del niño y del adolescente 2002; 1: 50-4.

21. Poyato Domínguez JL, Sánchez Espejo M, Cañete Estrada R, Poyato Domínguez M. Conductas anoréxicas en adolescentes de la ciudad de Córdoba. Rev Esp Pediatr 2002; 58: 241-6.

22. Toro i Trallero J, Farré C. Trastornos de la conducta alimentaria: anorexia y bulimia nerviosa. En: Prandi F. Programa de formación continuada en Pediatría Práctica. Barcelona: Prous Science, 2000; p.125-4.

23. Oliveros Calvo S. La adolescencia: un terreno abonado para las toxicomanías: Med Clin (Barc) 1995; 104: 777-9.

24. Shrier LA, Harris SK, Sternberg M, Beardslee WR. Associations of depression, self-esteem, and substance use with sexual risk among adolescents. Prev Med 2001; 33: 179-89

25. Joiner TE Jr. Pfaff JJ, Acres JG. Characteristic of suicidal adolescent and young adults presenting to primary care with non-suicidal (indeed nonpsychological) complaints. Eur J Public Health 2002; 12: 177-9.

26. Osona Rodríguez B, González Vicent M, Mencía Bartolomé S, Casado Flores J. Suicidio en la edad pediátrica: presentación de 30 casos. An Esp Pediatr 2000; $52: 31-5$

27. Aalto-Setälä T, Marttunen M, Tuulio-Henriksson A, Poikolainen K; Lönnqvist J. Depressive symptoms in adolescence as predictors of early adulthood depressive disorders and maladjustment. Am J Psychiatry 2002; 159: 1235-7.
28. Suicide and suicide attemps in adolescents. Committee on Adolescents. American Academy of Pediatrics. Pediatrics 2000; 1105: 871-4.

29. Elster AB, Kuznets NJ. Fundamentos y recomendación: Depresión (grave o recurrente) y suicidio. En: Guía de la AMA para actividades preventivas en el adolescente (GAPA). Recomendaciones y fundamentos. Elster AB, Kuznets (eds.). Madrid: Díaz de Santos; 1995.p. 147-65.

30. Whooley MA, Avins AL, Miranda J, Browner WS. Case-finding instruments for depression. Two questions are as good as many. J Gen Intern Med 1997; 12: 439-45.

31. Valdés Rodríguez J, Bolufer Vigrana N. Fracaso escolar en el adolescente. Perdiatr. Integral 1997; 2 : 272-84.

32. Argemí Renóm J. El pediatra ante el fracaso escolar. Archivos de Pediatría 1995; 46 Suplemento 1: $50-4$

33. Documento de Bases para una Ley de Calidad de la Educación. Madrid: Ministerio de Educación, Cultura y Deporte; 2002.

34. Elster AB, Kuznets NJ. Fundamentos y recomendación: Trastornos primarios del aprendizaje. En: Guía de la AMA para actividades preventivas en el adolescente (GAPA). Recomendaciones y fundamentos. Elster AB, Kuznets (eds.). Madrid: Díaz de Santos; 1995. p. 171-80.

35. Polaino-Lorente A. Fracaso escolar e hiperactividad. Archivos de Pediatría 1995; 46 Suplemento 1: 50-4.

36. James WH, West C, Deters KE, Armijo E. Youth dating violence. Adolescence 2000; 35: 455-65.

37. Vermeiren R, Ruchkin V, Leckman PE, Deboutte D, Schwab-Stone M. Exposure to violence and suicide risk in adoelscents: a community study. J Abnorm Child Psicol. 2002; 30: 529-37.

38. Irwin CE, Burg SJ, Uhler Cart C. America's adolescents: where have we bee, where are we going? J Adolesc Health 2002; 31: 91-121.

39. Berenson AB, Wiemann CM, Mc Combs S. Exposure to violence and associated health-risk behaviours among adolescent girls. Arch Pediatr Adolesc Med 2001; 155: 1238-42.

40. Vermeiren R, Schwab-Stone M, Ruckin VV, King RA, Van Heeringen C. A suicidal behavior and violence in male adolescents: a school-based study. J Am Acad Child Adolesc Psychiatry 2003; 42: 4-8. 
41. Flannery RB Jr. Preventing youth violence: a CISM pre-incident approach. Int J Emerg Ment Health 2000; 2: 167-70.

42. Sege R, Stringham P, Short S, Griffith J. Ten years after: examination of adolescent screening questions that predict future violence-related injury. J Adolesc Health 1999; 24. 395-402.

43. Comín E, Nebot M, Villalbí JR. Factors de risc lligats a l'estil de vida en l'edad evolutiva. El projecte FRISC de Barcelona. Barcelona: Publicacions de l'Ayuntament de Barcelona; 1989.

44. Elster AB, Kuznets NJ. Fundamentos y recomendación: Hábitos dietéticos, alteraciones de la alimentación y obesidad. En: Guía de la AMA para actividades preventivas en el adolescente (GAPA). Recomendaciones y fundamentos. Elster AB, Kuznets (eds.). Madrid: Díaz de Santos; 1995. p. 45-63.

45. American Psychiatric Association: Diagnostic and statistical manual of mental disorders, ed 4, DSMIV, Washington DC: American Psychiatric Association; 1994.

46. Wiederman MW, Prior T. Substance use among women with eating disorders. Int J Eat Disord 1996; 20: 163-8.
47. Trastornos del comportamiento alimentario. En: Psiquiatría de la adolescencia. Toro J. Madrid: Aula Médica Ediciones; 1999. p. 56-72.

48. Pla C, Toro J. Anorexia nervosa in Spanish adolescent sample: an 8 years longitudinal study. Acta Psychiatr Scand 1999; 100: 44-6.

49. Burke JD, Burke KC, Rae DS. Increased rates of drug abuse and dependence after onset of mood or anxiety disorders in adolescence. Hosp Community Psychiatry 1994; 45: 451-5.

50. Pereiro Berenguer I, Chover Lara JL, Salazar Cifré A, Roig Sena J, Gil Alcani J, Cordero Garrido I et al. Fracaso escolar en una cohorte de adolescentes. Atención Primaria 1999; 5: 289-95.

51. Mardomingo Sanz MJ. Agresividad y violencia en la infancia. Pediatr Integral 1999; número especial 2: $19-21$.

52. ¿Es la violencia escolar un problema emergente en la infancia?. En: Informe SIAS. Ponce Blandón J, Muriel Fernández R, Gómez de Terreros Sánchez I (eds.). Sevilla: Sección de Pediatría Social. An Esp Pediatr; 1997. p. 76-82.

53. Gómez de Terreros Sánchez I, Belinchón Sánchez C. Delincuencia y drogadicción en el niño y adolescente. Pediatr Integral 2001; 6: 107-32. 